\title{
EUS correlates of disconnected pancreatic duct syndrome in walled-off necrosis
}

Authors

Institutions
Ji Young Bang ${ }^{1}$, Udayakumar Navaneethan², Muhammad K. Hasan², Robert H. Hawes², Shyam Varadarajulu²

Indiana University, Indianapolis, Indiana, United States

Center for Interventional Endoscopy, Florida Hospital, Orlando, Florida, United States submitted 6. March 2016 accepted after revision 13. June 2016

\section{Bibliography}

Dol http://dx.doi.org/ 10.1055/s-0042-112586 Published online: 9.8.2016 Endoscopy International Open 2016; 04: E883-E889

(c) Georg Thieme Verlag KG Stuttgart · New York E-ISSN 2196-9736

\section{Corresponding author} Shyam Varadarajulu, MD Center for Interventional Endoscopy

Florida Hospital 601 East Rollins Street Orlando, FL 32803 Phone: +1-407-303-2570 Fax: +1-407-303-2585 svaradarajulu@yahoo.com
Background and study aims: Although the diagnostic features of disconnected pancreatic duct syndrome (DPDS) by computed tomography (CT) and magnetic/endoscopic retrograde cholangiopancreatography (MRCP/ERCP) have been established, no such characterization exists for endoscopic ultrasound (EUS). This study describes the imaging features of EUS that accurately define DPDS.

Patients and methods: This is a prospective study comprising 21 of 42 patients who underwent EUS-guided drainage of walled-off necrosis (WON) over an 18-month period. Findings on EUS were correlated with CT and pancreatography or surgical pathology when available. DPDS by EUS was defined by the presence of a well-defined fluid collection along the course of the main pancreatic duct with the upstream pancreatic parenchyma and duct terminating into the fluid collection. The main outcome measure was to as-

\section{Introduction}

\section{$\nabla$}

Disconnected pancreatic duct syndrome (DPDS) occurs in nearly $50 \%$ of patients with walled-off necrosis (WON) and is characterized by complete disruption of the main pancreatic duct, resulting in a variable portion of the upstream pancreatic gland becoming isolated from the main pancreatic duct downstream. DPDS can also occur as a result of chronic pancreatitis or after pancreatic surgery [1]. The disconnected pancreatic segment remains functional and in the absence of acinar atrophy, results in a persistent peripancreatic fluid collection (PFC), pancreatic ascites or nonresolving pancreaticocutaneous fistula $[2,3]$. The three criteria that best define DPDS are: 1 ) necrosis of at least $2 \mathrm{~cm}$ of the pancreas; 2 ) total cut-off the main pancreatic duct at the site of necrosis; and 3) viable distal pancreatic tissue upstream (towards the tail) from the site of necrosis [4]. sess the accuracy of EUS in diagnosing DPDS by correlation with CT and pancreatography or surgical pathology.

Results: Twenty-one patients with WON (median age 55 years; 15 males) constituted the study cohort. Median duration of pancreatitis was 12 weeks (range 5-20) and median WON size was $120 \mathrm{~mm}$ (range $40 \mathrm{~mm}$ to $200 \mathrm{~mm}$ ). At EUS, the upstream pancreatic parenchyma and duct were found to terminate within the WON in all $21 \mathrm{pa}-$ tients in whom DPDS was subsequently confirmed by follow-up CT in all patients, by ERCP in 17 , EUS-pancreatogram in 3 and surgical pathology in 1 .There was $100 \%$ correlation between EUS characterization of DPDS with CT and pancreatography or surgical pathology.

Conclusions: We report EUS findings indicating the presence of DPDS. These findings may have significant clinical implications for the management of patients with WON.

The most common modalities used for diagnosing DPDS are contrast-enhanced computed tomography (CECT) of the abdomen, magnetic resonance cholangiopancreatography (MRCP) and endoscopic retrograde cholangiopancreatography (ERCP). Early recognition of DPDS during the course of acute pancreatitis is important because a delay in diagnosis can lead to increased morbidity, prolonged hospital stay, and health-related costs. In a retrospective study of 26 patients with surgically proven DPDS, the average time to establishing the correct diagnosis from time of clinical presentation with a non-resolving PFC was 9.3 months [2]. Although pathognomonic features have been described for diagnosing DPDS by CECT [2], false-positive results are frequently reported in the setting of partial duct obstruction and an overlying PFC. While MRCP with secretin has emerged as a useful, noninvasive tool to assess pancreatic ductal integrity, the sensitivity of the technique for demonstrating extravasation at the 
site of pancreatic duct disconnection is less than that of ERCP [3, 5]. Additionally, in the setting of acute pancreatitis, the surrounding PFC oftentimes obliterates the view of the pancreatic duct. The diagnosis of DPDS can be reliably established at ERCP if there is extravasation of injected contrast from the main pancreatic duct without filling of the disconnected segment; however, the technique can potentially contaminate sterile collections, creating infected necrosis [6,7]. Furthermore, duodenal inflammation in the setting of severe acute pancreatitis can make the identification of the major papilla difficult and selective cannulation of the main pancreatic duct challenging.

Expectant management with enteral nutrition, antibiotics and anti-secretory therapy is the general norm for managing DPDS in the acute setting. However, the majority of WON in the setting of DPDS do not respond to conservative measures and require more definitive treatment. The 3 most common treatment strategies are percutaneous, endoscopic, and surgical (usually performed in conjunction with distal pancreatectomy) drainage techniques. Percutaneous drainage may lead to creation of longstanding pancreaticocutaneous fistula and surgery is associated with significant morbidity and mortality [8]. As a result, endoscopy is increasingly being utilized as a minimally invasive technique for internal transmural drainage of the PFC, usually under endoscopic ultrasound (EUS)-guidance. The technique involves the creation of a fistula between the gastrointestinal tract and the PFC, followed by stent placement. The transmural stents are usually removed after resolution of the PFC. However, in the setting of DPDS, this approach is associated with PFC recurrence rates as high as $50 \%[9,10]$ and leaving the plastic stents in situ permanently could prevent recurrence by creating a permanent fistula between the main pancreatic duct and the gastrointestinal tract $[11,12]$. Traditionally after endoscopic transmural drainage, PFC resolution is confirmed by CECT at 6 weeks to 8 weeks and the patient undergoes an ERCP or MRCP to assess for ductal integrity. If DPDS is confirmed, the transmural plastic stents are left in situ indefinitely. If the main pancreatic duct is intact, the stents are removed by endoscopy. This approach however, subjects patients to an additional endoscopic intervention. Of late, lumenapposing stents are being used with increasing frequency for PFC drainage [13]. It is postulated that the large diameter (10 $\mathrm{mm}$ to $15 \mathrm{~mm}$ ) of these stents provides better drainage but tissue overgrowth or ingrowth after breakdown of the plastic coating can imbed the stent in the retroperitoneum and thus, they cannot be left in situ indefinitely.

Our hypothesis is that if DPDS can be diagnosed reliably at time of EUS-guided transmural drainage of PFCs, it would enable the selection of appropriate stents (plastic not metal) for placement, preclude the need for follow-up MRCP or ERCP to assess for ductal integrity, and spare a subsequent endoscopy for stent removal. Therefore, the objective of the current study was to identify and describe the imaging features of EUS that accurately define DPDS.

\section{Patients and methods}

$\nabla$

Patients

This is a prospective observational study of patients with WON who were evaluated for participation in the Minimally Invasive Surgery versus Endoscopy Randomized (MISER) Trial (NCT02084537) and underwent EUS-guided transmural drainage of WON. Included in the study were patients aged $>18$ years with WON measuring $>6 \mathrm{~cm}$ and presenting with persistent abdominal pain, progressive clinical deterioration despite maximum medical support, gastric outlet obstruction or presence of infection manifesting as fever, sepsis or gas within the WON on CECT or magnetic resonance imaging (MRI). WON was categorized based on the revised Atlanta classification [14]. Excluded were patients with thrombocytopenia (platelet count $<50 \times$ $10^{9} / \mathrm{L}$ ), coagulopathy (INR $>1.5$ ) or those unable to undergo anesthesia.

All patients or their legally authorized representatives provided written informed consent for undergoing the procedures and participation in the clinical trial. The study protocol was approved by the Florida Hospital Institutional Review Board (IRB \# 577235; 754065). All patients had a minimum follow-up of 90 days.

\section{Procedural Technique}

All procedures were performed using a therapeutic linear array echoendoscope (GF UCT180T, Olympus America Inc., Center Valley, PA) under general or monitored anesthesia care. The linear array echoendoscope was advanced to the second portion of the duodenum and slowly torqued to identify the ventral pancreas. Once the pancreatic duct was identified, the echoendoscope was gradually withdrawn so as to trace the duct to the neck, body, and tail of the pancreas. If a WON was identified in the parenchyma during the tracing of the main pancreatic duct, its size, location, and relationship to the main pancreatic duct were documented. The upstream pancreas (towards the tail) was then identified using the celiac artery, spleen, and left kidney as anatomical landmarks and the pancreatic duct in the tail was traced to the genu of the pancreas. During tracing of the upstream duct, if an intervening WON was identified in the pancreatic parenchyma, the duct-WON relationship was documented. DPDS by EUS was defined by the presence of a well-defined fluid collection along the course of the main pancreatic duct with the upstream pancreatic parenchyma and duct terminating into the fluid collection ( Video 1 and $\bullet$ Video 2; $\bullet$ Fig. 1a, $\bullet$ Fig.1b $\bullet$ Fig. 1c, , $\odot$ Fig. 1d, and $\odot$ Fig.2). Patients subsequently underwent EUS-guided drainage of WON using a lumen-apposing metal stent or plastic stents.

\section{Follow-up Protocol}

A follow-up CECT of the abdomen was obtained at 8 weeks in all patients to assess the size of WON and hence response to treatment, and to identify the disconnected upstream pancreatic segment. If the WON had resolved completely, patients underwent an ERCP to assess for main pancreatic duct integrity ( Video 1; - Fig. 1d). If ERCP was unsuccessful, an EUS-guided pancreatogram was obtained by puncturing the upstream pancreatic duct using a $22 \mathrm{G}$ needle ( Video 3; $\bullet$ Fig. 3). If DPDS was confirmed, the transmural plastic stents were left in situ indefinitely in order to provide a conduit for drainage of the disconnected upstream pancreatic duct and prevent recurrence of WON. For patients with indwelling lumen-apposing metal stents, the metal stent was exchanged for 2 7Fr 4-cm double pigtail plastic stents. For patients with an intact main pancreatic duct, all transmural stents were removed because unlike in patients with DPDS, an additional conduit for drainage of the pancreatic duct is not needed and also because of the small risk of migration of the transmural stents [15]. Transpapillary stent placement into the main pancreatic duct was not performed in any patient with DPDS. 

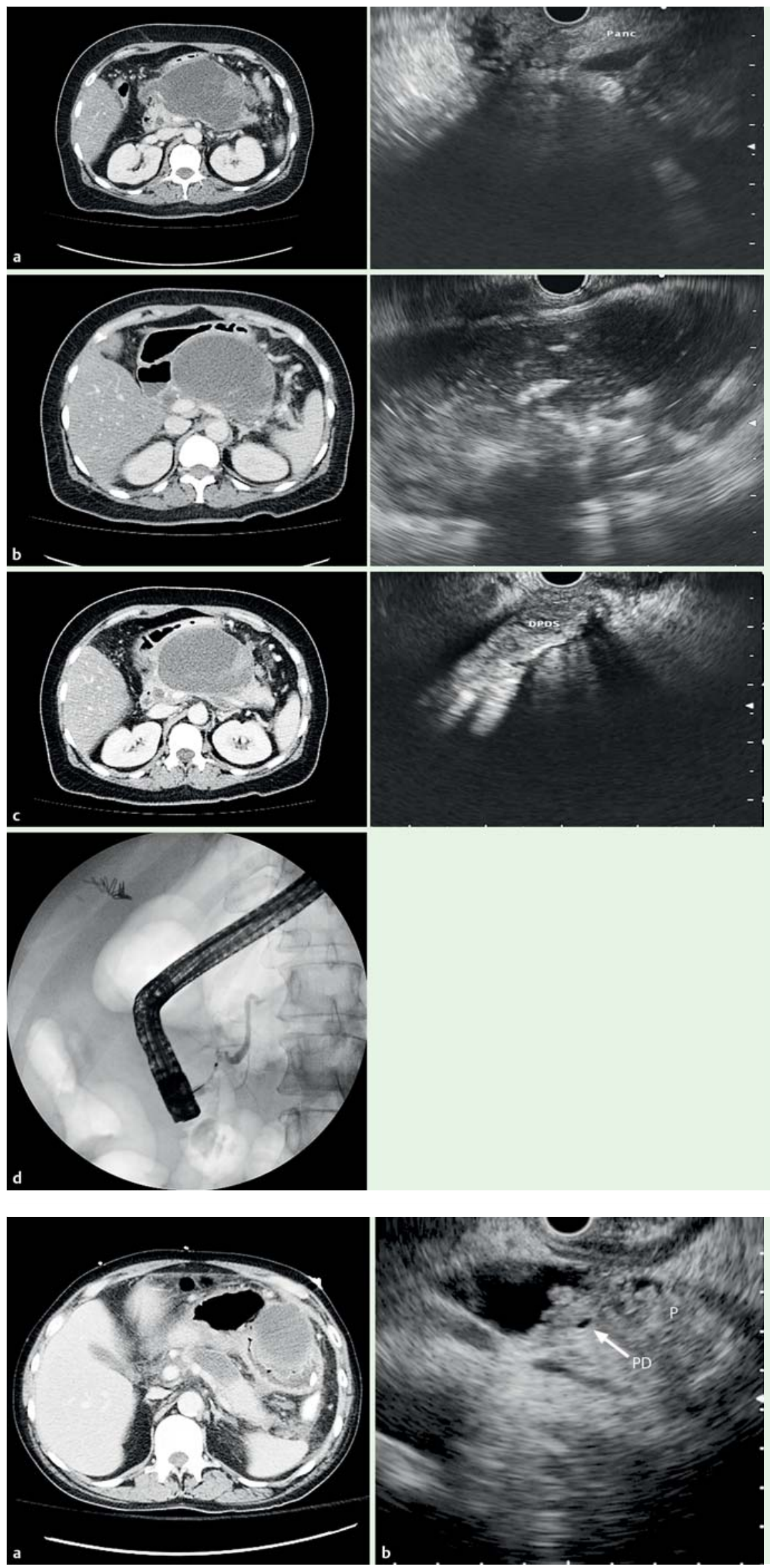

Fig. 1 a EUS and CT correlation of the pancreatic head region in a patient with DPDS and WON. $\mathbf{b}$ EUS and CT correlation of WON in the pancreatic body. c EUS and CT demonstrating the upstream disconnected pancreatic segment. $\mathbf{d}$ Demonstration of DPDS by pancreatogram at ERCP.
Fig. 2 EUS and CT correlation of the upstream main pancreatic duct draining into the WON. The non-dilated $1 \mathrm{~mm}$ pancreatic duct is better appreciated on EUS than with CT (Abbreviations: P, pancreas; PD, pancreatic duct). 
Table 1 Demographics, disease and PFC characteristics in 21 patients with disconnected pancreatic duct syndrome.

\begin{tabular}{|c|c|c|c|c|c|c|c|c|c|}
\hline No. & $\begin{array}{l}\text { Age } \\
\text { (yrs) }\end{array}$ & Gender & Etiology & $\begin{array}{l}\text { Pancreatitis } \\
\text { duration (weeks) }\end{array}$ & $\begin{array}{l}\text { WON size } \\
\text { (long axis, mm) }\end{array}$ & $\begin{array}{l}\text { WON size (short } \\
\text { axis, mm) }\end{array}$ & $\begin{array}{l}\text { WON } \\
\text { location }\end{array}$ & $\begin{array}{l}\text { Modality for DPDS } \\
\text { confirmation }\end{array}$ & $\begin{array}{l}\text { DPDS } \\
\text { location }\end{array}$ \\
\hline 1 & 79 & $M$ & Gallstones & 5 & 100 & 80 & Neck & ERCP & Neck \\
\hline 2 & 26 & $\mathrm{~F}$ & Gallstones & 7 & 90 & 80 & Body & ERCP & Body \\
\hline 3 & 54 & M & Alcohol & 10 & 70 & 50 & Neck & ERCP & Neck \\
\hline 4 & 64 & M & Gallstones & 8 & 120 & 110 & Body & ERCP & Neck \\
\hline 5 & 45 & M & Alcohol & 9 & 130 & 40 & Tail & ERCP & Body-Tail Jn. \\
\hline 6 & 48 & M & Alcohol & 6 & 150 & 50 & Body-Tail & ERCP & Body \\
\hline 7 & 74 & M & Idiopathic & 10 & 130 & 80 & Body & ERCP & Body \\
\hline 8 & 47 & $\mathrm{~F}$ & Gallstones & 12 & 90 & 80 & Body & ERCP & Body \\
\hline 9 & 39 & M & Alcohol & 12 & 160 & 150 & Head-Neck & ERCP & Neck \\
\hline 10 & 54 & M & Alcohol & 13 & 90 & 40 & Body-Tail & ERCP & Body \\
\hline 11 & 68 & M & Gallstones & 13 & 130 & 80 & Body & ERCP & Body \\
\hline 12 & 55 & M & Gallstones & 15 & 100 & 80 & Body-Tail & ERCP & Body \\
\hline 13 & 45 & M & Alcohol & 13 & 200 & 180 & Body-Tail & ERCP & Body-Tail Jn. \\
\hline 14 & 57 & $M$ & Alcohol & 13 & 130 & 80 & Body & ERCP & Body \\
\hline 15 & 65 & $M$ & Idiopathic & 13 & 40 & 40 & Body & ERCP & Body \\
\hline 16 & 65 & $\mathrm{~F}$ & Idiopathic & 15 & 50 & 40 & Head-Neck & Surgery & Neck \\
\hline 17 & 34 & M & Gallstones & 16 & 150 & 100 & Body-Tail & ERCP & Body \\
\hline 18 & 69 & $\mathrm{~F}$ & Post-ERCP & 20 & 120 & 80 & Body & EUS & Neck \\
\hline 19 & 51 & M & Alcohol & 9 & 80 & 65 & Neck & EUS & Neck \\
\hline 20 & 56 & $\mathrm{~F}$ & Idiopathic & 8 & 50 & 50 & Neck & EUS & Neck \\
\hline 21 & 67 & $\mathrm{~F}$ & Idiopathic & 8 & 130 & 80 & Body & ERCP & Neck \\
\hline
\end{tabular}

DPDS, disconnected pancreatic duct syndrome; ERCP, endoscopic retrograde cholangiopancreatography; EUS, endoscopic ultrasound; F, female; Jn, junction; M, male; $\mathrm{mm}$; millimeters; WON, walled-off necrosis-

Information on patient demographics, etiology and duration of pancreatitis, findings on follow-up CT, EUS, pancreatography and surgical pathology when available were documented.

\section{Main Outcome Measures}

The main outcome measure was to assess the accuracy of EUS in diagnosing DPDS by correlation with findings on follow-up CT, pancreatogram or surgical pathology.

\section{Statistical Analysis}

Patient characteristics, PFC features, and procedure details were summarized. Categorical data were expressed as frequencies and percentages, whereas continuous data were summarized as medians with range. The dataset was compiled using Microsoft Excel (Microsoft Corporation, Redmond, WA) and all statistical analyses were performed using Stata 14 (StataCorp LP, College Station, TX).

\section{Results}

42 patients underwent EUS-guided drainage of WON during an 18-month period from May 2014 to November 2015. Of the 42 patients, 21 were excluded as EUS characterization of the pancreatic tail/upstream gland was suboptimal in 9, follow-up pancreatogram was not available in 8 , WON was extra-pancreatic in 3 and secondary to pancreatic cancer in 1 .The remaining 21 patients (median age 55 years [range 26 - 79 years]; 15 males) constituted the study cohort. Details of patient demographics, clinical information and WON characteristics are shown in $\bullet$ Table 1. Alcohol (38.1\%) and gallstones (33.3\%) were the most common etiologies and the median duration of pancreatitis was 12 weeks (range 5 weeks to 20 weeks). The median size of the WON on CT/ MRI in the long axis was $120 \mathrm{~mm}$ (range $40 \mathrm{~mm}$ to $200 \mathrm{~mm}$ ) and were predominantly located in the pancreatic head-neck in 2 , neck in 4 and the body-tail region in 15 patients. Eleven of 21 patients underwent EUS-guided drainage of WON more than 10 weeks after the inciting episode of acute pancreatitis due to the referral pattern in some patients and poor encapsulation of the PFC even beyond 8 weeks that were not suitable for intervention in others. At EUS, the upstream pancreatic parenchyma and duct were found to terminate into the WON in all 21 patients. Six of the 21 patients also underwent placement of a percutaneous drain for lavage of the necrotic contents.

On clinical and radiological follow-up, 20 patients had disease resolution and 1 had disease progression requiring surgical necrosectomy with distal pancreatectomy. Follow-up CT revealed a viable remnant pancreas upstream from the site of transmural drainage (evident by the presence of transmural stents) in the other 20 patients. Obvious pancreatic ductal dilation was observed in the disconnected segment in only one of 20 patients ( $\bullet$ Fig.3). Pancreatogram at ERCP was successful in 17 of 20 patients and failed in 3; EUS-guided pancreatogram was successful in all 3 patients in whom ERCP failed. None of the patients who underwent EUS-guided pancreatogram experienced adverse events as only a $22 \mathrm{G}$ needle was used for puncture and rectal indomethacin was administered in all patients as a prophylactic measure. DPDS was identified at ERCP or EUS-guided pancreatogram in all 20 subjects. Specimen examination of the patient who underwent distal pancreatectomy confirmed the presence of DPDS.Complete disconnection of the duct was identified in the body of the pancreas in 10 patients, neck of the pancreas in 9 and body-tail junction in 2 . There was $100 \%$ correlation between EUS characterization of DPDS with CT, pancreatography or surgical pathology in all 21 patients. The transmural stents were left in situ in 20 patients and at a median follow-up of 272 days (range 68-501 days), none of the patients developed PFC recurrence or reported major disease-related symptoms at follow-up. 


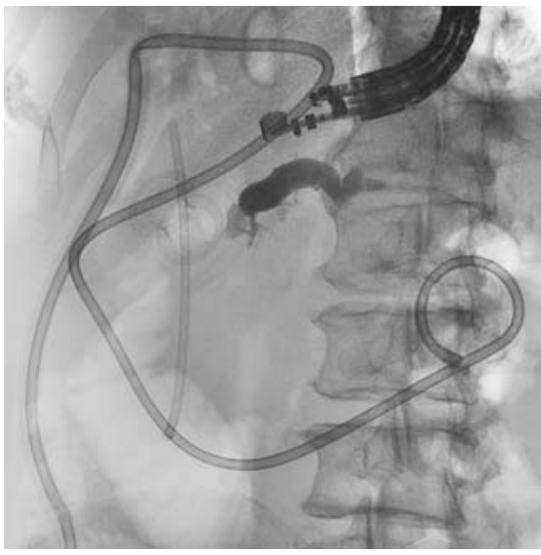

Fig. 3 EUS-guided pancreatogram demonstrating DPDS

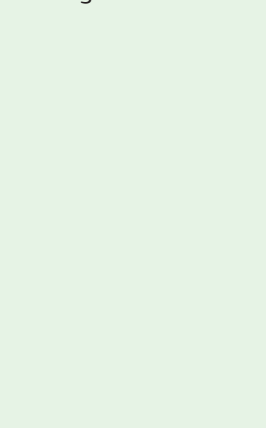

\section{Discussion}

When a well-defined fluid collection is observed at EUS along the course of the main pancreatic duct with the upstream pancreatic parenchyma and duct terminating into the fluid collection, it reliably correlates with CT and ERCP findings of DPDS. We believe that this observation at EUS is clinically relevant as it facilitates early recognition of the disease and helps to make management decisions critical to treatment outcomes.

DPDS is associated with significant long-term morbidity because of continued unawareness of the disease, delayed diagnosis, lack of expertise, and inability to provide appropriate and timely multidisciplinary interventions [3]. Despite multidisciplinary management, some patients may develop a persistent internal or external fistula due to a non-resolving PFC that cannot drain via the natural orifice (ampulla), diabetes due to atrophy of the disconnected segment, or secondary portal hypertension due to thrombosis of the portal vein. The ability to recognize DPDS and intervene early is key to treatment success. Given the high morbidity ( $14 \%$ to $20 \%$ ) and mortality ( $1 \%$ to $20 \%$ ) for surgery when undertaken in the acute setting in debilitated patients, there is an increased focus on non-operative treatment of DPDS adopting endoscopic techniques [16-18]. Endoscopic strategies aimed at treating pancreatic duct disruption are pancreatic sphincterotomy, transpapillary stent or drain placement, and endoscopic transmural drainage of the PFC. However, despite being widely practiced, pancreatic sphincterotomy and transpapillary stenting have minimal or no role in the management of DPDS. These techniques are more useful in patients with partial disruptions when the site of leak can be bridged [19]. Transmural drainage, on the other hand, is not dependent on the existence of a patent communication between the normal proximal duct and the discon- nected upstream segment. The fistulous communication by means of a cystenterostomy therefore enables adequate drainage of the disconnected gland.

In our opinion, DPDS is best managed when patients present with a mature and "reasonable-sized" WON. If WON is larger than $4 \mathrm{~cm}$ in its largest dimension and located within $15 \mathrm{~mm}$ of the gastrointestinal lumen, EUS-guided internal drainage is straight-forward and safe ( Video 4 ). It is also important to leave the transmural stents in situ indefinitely as the permanent pancreaticogastric/duodenal fistula prevents PFC recurrence. If the stents are removed prematurely or inadvertently based on suboptimal cross-sectional imaging findings, then following the closure of the transmural tract, the upstream gland drains via an external fistula or progresses to form a non-resolving, small yet symptomatic PFC. These PFCs may require very complex treatment measures, such as a combined rendezvous approach using endoscopic and percutaneous techniques to direct the pancreatic secretion back into the intestinal lumen [8]. Current practice at most institutions is to perform an MRCP or CT scan to assess the resolution of the WON and then perform an ERCP to access the status of the main pancreatic duct. The limitations of this strategy are:1) both CT and MRCP are unreliable in discerning a partial from complete duct disruption if a PFC overlies the main pancreatic duct; 2) the sensitivity of MRCP is lower than that of ERCP because the intra-ductal pressure produced by manual injection of the contrast during ERCP cannot be matched by MRCP, even with the increased exocrine output in response to secretin; 3) the presence of an indwelling endoprosthesis makes assessment of ductal integrity by cross-sectional imaging more challenging particularly after resolution of the WON; and 4) with increasing use of the lumen-apposing metal stents for PFC drainage, MRCP cannot be performed in a substantial proportion of the patients.

So how can the observations from this study positively influence management of DPDS in the setting of WON? In patients with WON referred for EUS-guided drainage, if the diagnosis of DPDS can be reliably established during the index EUS examination, plastic rather than metal stents should be placed for internal drainage. These stents must be left in situ indefinitely and a repeat endoscopy for stent removal is not required. If the WON is very large and a multi-gate drainage technique using lumen-apposing metal stents is adopted, then at least 1 of the gateways must be specifically created using plastic stents so that they can be left in situ indefinitely ( Video 5; $\bullet$ Fig.4a, $\bullet$ Fig.4b). In addition, an ERCP to exclude DPDS, which is often technically challenging to perform in this patient population, may not be required in a significant proportion of patients. Fifty percent ( 21 of 42 WON patients) in our study cohort may not have required a follow-up ERCP based on the EUS findings. Finally, this strategy is
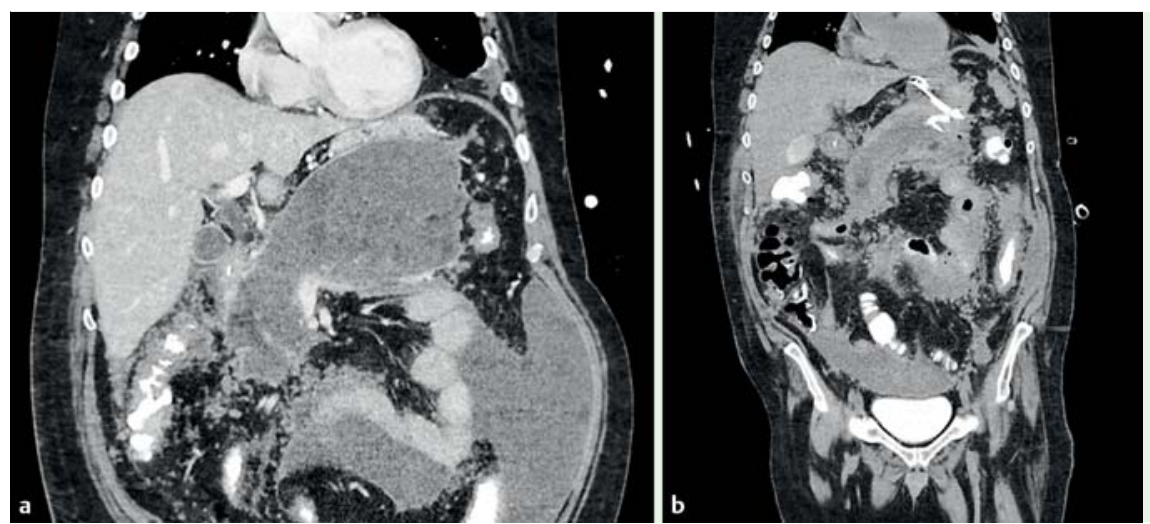

Fig. 4 a Coronal CT image revealing a large communicating WON measuring $200 \times 180 \mathrm{~mm}$. b After treatment using the multi-gate technique where a lumen-apposing metal stent was placed into one transmural tract and two plastic stents were inserted into the second tract, a follow-up CT at 72 hours revealed a $75 \%$ decrease in the volume of the collection. While the lumen-apposing metal stent was removed, the plastic stents were left in situ indefinitely to drain the upstream disconnected gland. 


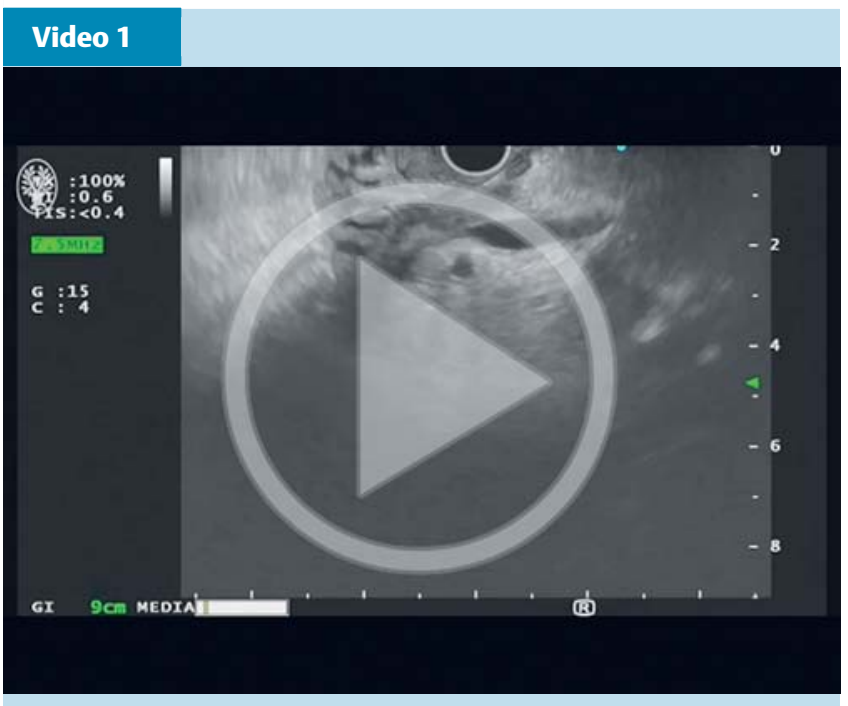

Demonstration of DPDS by EUS with CT and ERCP correlation. Online content including video sequences viewable at: http://dx.doi.org/10.1055/s0042-112586

\section{Video 2}

(20.

Disconnected pancreatic duct syndrome Complete disruption of the main PD resulting in a portion of the upstream pancreatic gland becoming isolated from the main $\mathrm{PD}$ downstream

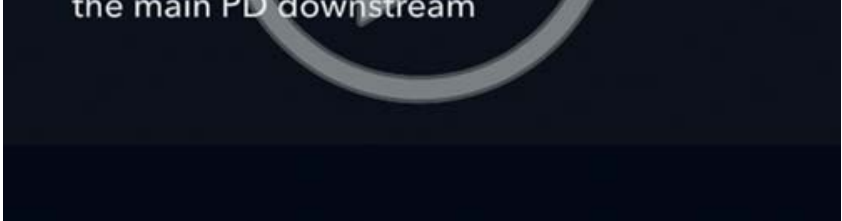

Demonstration of the disconnected (upstream) pancreatic duct and parenchyma draining into the fluid collection. Online content including video sequences viewable at: http://dx.doi.org/10.1055/s-0042-112586

likely to minimize the incidence of PFC recurrence in the setting of DPDS and the challenges associated with its treatment.

There are several limitations to the current study. First, this is a prospective observational study involving a small cohort of 21 patients and the data on the technical or clinical outcomes of the endoscopic interventions are not presented. However, the objective of our study was solely to develop EUS correlates of DPDS and not to evaluate treatment outcomes. Second, we observed that EUS was effective only for evaluating WON and duct disruptions in the neck-body-tail regions of the pancreas. Once the echoendoscope is advanced to the duodenum, the complex anatomy of the pancreatic head region makes it difficult to study the relationship between the WON and main pancreatic duct. Likewise, if the WON is very large, it is technically difficult to examine the pancreatic tail region for the presence of viable parenchyma or duct. The mechanical compression induced by the WON obscures sonographic landmarks, making examination more challenging,

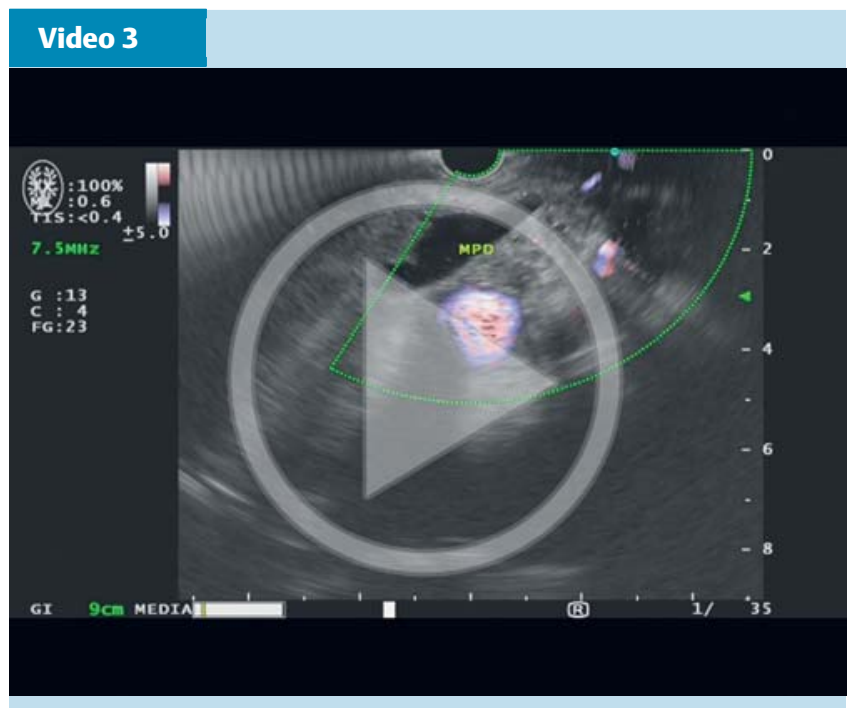

EUS-guided pancreatogram demonstrating DPDS. Online content including video sequences viewable at: http://dx.doi.org/10.1055/s-0042-112586



EUS-guided drainage of WON in the setting of DPDS. Online content including video sequences viewable at: http://dx.doi.org/10.1055/s-0042-112586

and in fact, 9 patients undergoing EUS-guided WON drainage were excluded from the study due to suboptimal visualization of the pancreatic tail/upstream gland. Third, we did not compare the diagnostic performance of EUS with CECT or MRCP for diagnosing DPDS and that was not the intent of this study. In our experience, once the WON resolves, the associated residual inflammation and presence of endoprostheses make CECT and MRCP unreliable for diagnosing DPDS. In addition, the upstream ductal dilation described in the radiologic literature was reported in the chronic setting and hence was not observed in the majority of our patients. Finally, this report pertains to DPDS only in the setting of WON and not to chronic pancreatitis. Therefore, the relevance of these observations in other conditions such as pseudocysts and necrotizing interstitial pancreatitis is unclear. 




EUS-guided drainage of WON using the multi-gate technique in which one gateway is created using a lumen-apposing metal stent and the other using plastic stents. At 8-week follow-up, the lumen-apposing metal stent was removed and the plastic stents were left in situ indefinitely. Online content including video sequences viewable at: http://dx.doi.org/10.1055/s-0042112586

\section{Conclusions}

In conclusion, we report the EUS features of DPDS in the setting of WON. We believe that these findings may be important as they facilitate early recognition of the disease and help with informed decision-making about therapeutic interventions which, in turn, may have favorable impact on treatment outcomes.

Competing interests: Drs. Varadarajulu and Hawes are consultants for Boston Scientific Corporation and Olympus America, Inc.

\section{References}

1 Deviere J, Buesco H, Baize M et al. Complete disruption of the main pancreatic duct: endoscopic management. Gastrointest Endosc 1995; 42: $445-451$

2 Tann M, Maglinte D, Howard TJ et al. Disconnected pancreatic duct syndrome: imaging findings and therapeutic implications in 26 surgically corrected patients. J Comput Assist Tomogr 2003; 27: 577-582

3 Nadkarni NA, Kotwal V, Sarr MG et al. Disconnected Pancreatic Duct Syndrome. Endoscopic Stent or Surgeon's Knife? Pancreas 2015; 44: $16-22$
4 Nealon WH, Bhutani M, Riall TS et al. A unifying concept: pancreatic ductal anatomy both predicts and determines the major complications resulting from pancreatitis. J Am Coll Surg 2009; 27: 1389-1400

5 Gillams AR, Kurzawinski T, Lees WR. Diagnosis of duct disruption and assessment of pancreatic leak with dynamic secretin-stimulated MR cholangiopancreatography. Am J Roentgenol 2006; 186: 499-506

6 Banks PA, Freeman M. Practice Parameters Committee of the American College of Gastroenterology. Practice guidelines in acute pancreatitis. Am J Gastroenterol 2006; 101: 2379-2400

7 Trevino JM, Tamhane A, Varadarajulu S. Successful stenting in ductal disruption favorably impacts treatment outcomes in patients undergoing transmural drainage of peripancreatic fluid collections. J Gastroenterol Hepatol 2010; 25: 526-531

8 Irani S, Gluck M, Ross A et al. Resolving external pancreatic fistulas in patients with disconnected pancreatic duct syndrome: using rendezvous techniques to avoid surgery (with video). Gastrointest Endosc 2012; 76: 586-593

9 Lawrence C, Howell DA, Stefan AM et al. Disconnected pancreatic tail syndrome: potential for endoscopic therapy and results of long-term follow-up. Gastrointest Endosc 2008; 67: 673-679

10 Baron TH, Harewood GC, Morgan DE et al. Outcome differences after endoscopic drainage of pancreatic necrosis, acute pancreatic pseudocysts, and chronic pancreatic pseudocysts. Gastrointest Endosc 2002; 56: 7-17

11 Arvanitakis $M$, Delhaye M, Bali MA et al. Pancreatic-fluid collections: a randomized controlled trial regarding stent removal after endoscopic transmural drainage. Gastrointest Endosc 2007; 65: 609-619

12 Varadarajulu S, Wilcox CM. Endoscopic placement of permanent indwelling transmural stents in disconnected pancreatic duct syndrome: does benefit outweigh the risks? Gastrointest Endosc 2011; 74: $1408-1412$

13 Rinninella $E$, Kunda R, Dollhopf $M$ et al. EUS-guided drainage of pancreatic fluid collections using a novel lumen-apposing metal stent on an electrocautery-enhanced delivery system: a large retrospective study (with video). Gastrointest Endosc 2015; 82: 1039-1046

14 Banks PA, Bollen TL, Dervenis C et al. Classification of acute pancreatitis-2012: revision of the Atlanta classification and definitions by international consensus. Gut 2013; 62: 102-111

15 Varadarajulu S, Wilcox CM. Endoscopic placement of permanent indwelling transmural stents in disconnected pancreatic duct syndrome: does benefit outweigh the risks? Gastrointest Endosc 2011; 74: $1408-1412$

16 Howard TJ, Rhodes GJ, Selzer DJ et al. Roux-en-Y drainage is the best surgical option to treat patients with disconnected duct syndrome after severe acute pancreatitis. Surgery 2001; 130: 714-719

17 Howard JM. Surgical treatment of chronic pancreatitis. In: Howard JM, Jordan G, Reber H (eds.) Surgical Disease of the Pancreas. Philadelphia, PA: Lea and Febigeer; 1987: 3 496-521

18 Varadarajulu S, Rana SS, Bhasin DK. Endoscopic therapy for pancreatic duct leaks and disruptions. Gastrointest Endosc Clin N Am 2013; 23: $863-892$

19 Varadarajulu S, Noone TC, Tutuian $R$ et al. Predictors of outcome in pancreatic duct disruption managed by endoscopic transpapillary stent placement. Gastrointest Endosc 2005; 61: 568-575 